\title{
Peripapillary Retinal Nerve Fiber Layer Thickness Change After Panretinal Photocoagulation in Patients With Diabetic Retinopathy
}

\author{
Ho Young Kim, MD, Ho Kyun Cho, MD, PhD \\ Department of Ophthalmology, College of Medicine, Chung-Ang University Hospital, Seoul, Korea
}

\begin{abstract}
Purpose: To examine the effect of panretinal photocoagulation (PRP) on the retinal nerve fiber layer (RNFL) thickness in patients with diabetic retinopathy.

Methods: Subjects included 118 eyes for a treatment group and 164 eyes for a control group. The peripapillary RNFL thickness was measured before and 6 months after PRP in treatment group. In control group, the peripapillary RNFL thickness was measured at baseline and 6 months later. The relationships between changes in RNFL thickness and the number of laser burns, duration of diabetes, $\mathrm{HbA1c}$ level, and vision change were analyzed.

Results: After 6 months, the RNFL thickness decreased an average of $2.12 \mu \mathrm{m}$ and $0.93 \mu \mathrm{m}$ in the treatment and control groups. However, the changes between the two groups were not statistically significant. The relationship between the number of laser burns and changes in RNFL thickness was not significant. No differences were found between changes in the RNFL thickness and the duration of diabetes in either group. However, in the treatment group a higher $\mathrm{HbA} 1 \mathrm{c}$ level was correlated with a greater decrease in post-PRP RNFL thickness. This relationship was not observed in the control group. The difference in the change of the RNFL thickness between the two groups was statistically significant. Vision increased an average of 0.02 and 0.01 after 6 months in the treatment and control groups, respectively. However, this difference was not statistically significant.

Conclusions: Although a decrease in peripapillary RNFL thickness was observed in the treatment group after 6 months, it was not statistically significant compared to control group. However, the decrease was greater when the blood $\mathrm{HbA} 1 \mathrm{c}$ level was higher.
\end{abstract}

Korean J Ophthalmol 2009;23:23-26 (c) 2009 by the Korean Ophthalmological Society.

Key Words: Diabetic retinopathy, Glycosylated hemoglobin, Panretinal photocoagulation, Optical coherence tomography, Peripapillary retinal nerve fiber layer

Panretinal photocoagulation (PRP) reduces the risk of severe visual loss by $50 \%$ in patients with diabetic retinopathy. ${ }^{1}$ Most physicians agree that the intensity of a laser is enough to stimulate the outer retinal layer. ${ }^{2}$ If the laser intensity is too strong and destroys the entire retinal layer, it will cause a sequential decrease in the peripapillary retinal nerve fiber layer (RNFL) thickness. Also, it is reasonable to hypothesize that the peripapillary RNFL thickness should decrease in

Received: June 9, 2008 Accepted: January 21, 2009

Reprint requests to Ho Kyun Cho, MD, PhD. Department of Ophthalmology, College of Medicine, Chung Ang University Hospital \#224-1, Heukseok-dong, Dongjak-gu, Seoul 156-755, Korea. Tel: 82-2-6299-1685, Fax: 82-2-6299-1077, E-mail: hkcho26@cau.ac.kr

\footnotetext{
* This Research was supported by the Chung-Ang University Research Grants in 2007.

* This article was presented as a poster at the Association for Research in Vision and Ophthalmology annual meeting, April 27-May 1, 2008; Fort Lauderdale, Florida, USA.
}

proportion to the frequency of the laser burns. Most physicians perform PRP as a first line strategy in the treatment of diabetic retinopathy; however, no reports have investigated peripapillary RNFL thickness change following PRP.

The goal of this study is to evaluate the peripapillary RNFL thickness after PRP in patients with diabetic retinopathy. We also examined possible relationships between changes in peripapillary RNFL thickness and 1) HbAlc level, 2) diabetes duration, and 3 ) interval visual acuity.

\section{Materials and Methods}

The medical records of patients with diabetic retinopathy were reviewed retrospectively. The subjects were divided into a treatment group and a control group. The treatment group included patients who had received PRP. All patients in the treatment group had at least severe non-proliferative diabetic retinopathy and received PRP treatment. All patients received a moderate degree of laser burns from an argon laser photocoagulator (Coherent Ultima 2000, CA, USA). A laser 
Table 1. Baseline data of the treatment group and the control group

\begin{tabular}{lccc}
\hline & Treatment group & Control group & \\
\hline Number of eyes & 118 & 164 & \\
Age (mean, years) & $59.2 \pm 10.1$ & $56.1 \pm 9.8$ & 0.853 \\
Duration of diabetes (mean, years) & $12.6 \pm 6.2$ & $6.8 \pm 4.2$ & $<0.001^{*}$ \\
BCVA & $0.34 \pm 0.12$ & $0.86 \pm 0.14$ & $<0.001^{*}$ \\
\hline
\end{tabular}

$\mathrm{BCVA}=$ best corrected visual acuity.

$* p<0.05, t$-test was performed between the treatment group and the control group

Table 2. Interval changes of peripapillary RNFL thickness in the treatment and control groups

\begin{tabular}{lccccccc}
\hline & $\begin{array}{c}\text { Number of } \\
\text { eyes }\end{array}$ & $\begin{array}{c}\text { Number of } \\
\text { laser burns }\end{array}$ & $\begin{array}{c}\text { RNFL thickness } \\
\text { at baseline }(\mu \mathrm{m})\end{array}$ & $\begin{array}{c}\text { RNFL thicknes } \\
\text { at } 6 \text { months }(\mu \mathrm{m})\end{array}$ & $\begin{array}{c}\text { RNFL thickness } \\
\text { difference* }(\mu \mathrm{m})\end{array}$ & $p$ & $p^{\ddagger}$ \\
\hline Treatment group & 118 & $1576.5 \pm 415.7$ & $348.5 \pm 48.1$ & $346.4 \pm 53.4$ & $2.12 \pm 7.34$ & $<0.001^{\dagger}$ \\
Control group & 164 & no & $364.2 \pm 54.2$ & $363.3 \pm 58.1$ & $0.93 \pm 4.24$ & 0.127 & 0.31 \\
\hline
\end{tabular}

$\mathrm{RNFL}=$ retinal nerve fiber layer; $\mathrm{PRP}=$ panretinal photocoagulation.

*RNFL thickness at 6 months - RNFL thickness at baseline

${ }^{\dagger} p<0.05, t$-test was performed between baseline and 6 months

${ }^{*} p$ value, ANOVA was performed between the treatment group and the control group

Table 3. Interval changes of visual acuity in the treatment group and the control group

\begin{tabular}{lccccccc}
\hline & $\begin{array}{c}\text { Number of } \\
\text { eyes }\end{array}$ & $\begin{array}{c}\text { Number of } \\
\text { laser burns }\end{array}$ & $\begin{array}{c}\text { BCVA at baseline, } \\
\text { mean }\end{array}$ & $\begin{array}{c}\text { BCVA at } 6 \text { months, } \\
\text { mean }\end{array}$ & $\begin{array}{c}\text { Interval difference } \\
\text { of BCVA* }\end{array}$ & $p$ & $p^{*}$ \\
\hline Treatment group & 118 & $1576.5 \pm 415.7$ & $0.34 \pm 0.12$ & $0.36 \pm 0.25$ & $0.02 \pm 0.10$ & $<0.001^{\dagger}$ & 0.357 \\
Control group & 164 & no & $0.86 \pm 0.14$ & $0.85 \pm 0.23$ & $0.01 \pm 0.12$ & 0.248 & \\
\hline
\end{tabular}

$\mathrm{PRP}=$ panretinal photocoagulation; $\mathrm{BCVA}=$ best corrected visual acuity.

$*$ BCVA at 6 months - BCVA at baseline

${ }_{p}^{\dagger} p<0.05, t$-test was performed between baseline and 6 months

${ }^{\ddagger} p$ value, ANOVA was performed between the treatment group and the control group

burn of a moderate degree refers to a laser burn resulting in a hazy, mucoid, translucent, and foggy chorioretinal lesion. The control group included patients with moderate or mild non-proliferative diabetic retinopathy who did not receive laser treatment. Eyes which had any condition that could influence the peripapillary RNFL thickness were excluded from the study. The peripapillary RNFL thickness was measured before and 6 months after PRP in the treatment group. In the control group, the peripapillary RNFL thickness was measured at the baseline point of the study and 6 months later. The peripapillary RNFL thickness was measured with Stratus optical coherence tomography (OCT) 3 (Carl Zeiss Meditec, Dublin, CA, USA). Collected data included age, gender, duration of diabetes, level of blood glycosylated hemoglobin (HbA1C), total number of laser burns, and best corrected visual acuity. The final sample included 118 eyes (59 patients) in the treatment group and 164 eyes ( 82 patients) in the control group. A series of ANOVA and $t$-tests were conducted using SPSS to examine 1) a demographic difference between the two groups and 2) an interval change inthe peripapillary RNFL thickness in both groups. We also examined possible relationships between changes in peripapillary RNFL thickness and 1) HbAlc level, 2) diabetes duration, and 3) interval visual acuity in both groups.

\section{$\underline{\text { Results }}$}

Table 1 shows the demographic data of the 118 eyes from the 59 PRP-treated subjects and the 164 eyes of the 82 control subjects. There were no differences in age or gender between the two groups. However, in the treatment group the duration of diabetes was longer and the best corrected visual acuity was lower than in the control group. These differences were statistically significant $(p<0.001)$.

In the treatment group the post-PRP peripapillary RNFL thickness decreased an average of $2.12 \mu \mathrm{m}(348.5 \pm 48.1 \mu \mathrm{m}$ at baseline to $346.4 \pm 53.4 \mu \mathrm{m} 6$ months later, $p<0.001$ ), while an average decrease of $0.93 \mu \mathrm{m}(364.2 \pm 54.2 \mu \mathrm{m}$ at baseline to $363.3 \pm 58.1 \mu \mathrm{m} 6$ months later $p=0.127$ ) was observed in the control group (Table 2). However, the changes were not statistically different between the two groups ( $p=0.31$, Table 2). In addition, there was no significant relationship between the number of laser burns and changes in the peripapillary RNFL thickness in the treatment group ( $p=0.511$, ANOVA).

In the treatment group the post-PRP visual acuity increased an average of $0.02(0.34 \pm 0.12$ at baseline to $0.36 \pm 0.256$ months later, $p<0.001$, Table 3$)$. Despite a significant increase in visual acuity, there was no significant relationship between the number of laser burns and changes in visual acuity $(p=0.873$, ANOVA). In the control group, visual acuity at 6 months had 
Table 4. Comparison of interval changes of the peripapillary RNFL thickness according to changes in visual acuity

\begin{tabular}{|c|c|c|c|c|c|c|}
\hline \multirow{2}{*}{ Interval change of $\mathrm{BCVA}$} & \multicolumn{2}{|c|}{ Improved } & \multicolumn{2}{|c|}{ Unchanged } & \multicolumn{2}{|c|}{ Worsened } \\
\hline & Treatment group & Control group & Treatment group & Control group & Treatment group & Control group \\
\hline Number of eyes & 39 & 6 & 63 & 130 & 16 & 28 \\
\hline Change of RNFL thickness $(\mu \mathrm{m}) *$ & $2.21 \pm 8.27$ & $1.02 \pm 4.27$ & $1.99 \pm 7.92$ & $0.87 \pm 5.72$ & $2.18 \pm 7.92$ & $0.89 \pm 5.24$ \\
\hline$p$ & $<0.05^{\dagger}$ & 0.165 & 0.788 & 0.879 & 0.058 & $<0.001^{\dagger}$ \\
\hline$p^{\ddagger}$ & $>0.05$ & & $>0.05$ & & $>0.05$ & \\
\hline
\end{tabular}

$\mathrm{RNFL}=$ retinal nerve fiber layer; $\mathrm{PRP}=$ panretinal photocoagulation; $\mathrm{BCVA}=$ best corrected visual acuity.

$*$ RNFL thickness at 6 months - RNFL thickness at baseline

$p<0.05, t$-test was performed between baseline and 6 months

${ }^{*} p$ value, ANOVA was performed between the treatment group and the control group

decreased an average of 0.01 , but the change was not significant $(0.86 \pm 0.14$ at baseline to $0.85 \pm 0.23$ at 6 months, $p=0.248$, Table 3). There was no difference between the two groups regarding interval changes of visual acuity ( $p=0.357$, Table 3 ).

The subjects were divided into improved, unchanged, or worsened groups according to the interval changes of the visual acuity. Among the improved cases the treatment group showed a significant decrease of the peripapillary RNFL thickness $(p<0.05$, Table 4$)$. However, there was no significant difference between the two groups ( $p>0.05$, Table 4 ).

There was no significant relationship between changes in the peripapillary RNFL thickness and the duration of diabetes in either group ( $p>0.05$, ANOVA). However, in the treatment group a higher $\mathrm{HbAlc}$ level was correlated with a significantly greater decrease in post-PRP RNFL thickness ( $p<0.05$, ANOVA). This relationship was not observed in the control group.

\section{Discussion}

Although PRP has been proven to be an effective treatment strategy for severe DR, the laser intensity utilized is decided by the physician. Most physicians agree that laser intensity which causes damage to the entire retinal layer should be avoided. It has been reported that a high intensity laser beam can cause destruction of the entire retinal layer, including ganglion cells. ${ }^{3}$ Damage to ganglion cells results in loss of the retinal nerve fiber layer and a sequential decrease in the peripapillary nerve fiber layer thickness. ${ }^{4}$ In our study, the peripapillary RNFL thickness decreased in both the treatment and control groups and the difference between the two groups was not statistically significant. Therefore, it is difficult to conclude that the change in thickness was due to the laser treatment. Our study suggests that retinal laser photocoagulation, which can cause laser burns, does not necessarily damage the retinal nerve fiber layer if the laser burn is only of a moderate degree. However, the results are limited to 6 months of follow-up.

There was no relationship between laser burn frequency and peripapillary RNFL thickness change in the treatment group. This indicates that an adequate intensity of retinal laser photocoagulation does not alter retinal function in patients with DR.

Parikh et al. ${ }^{5}$ suggested that the retinal nerve fiber becomes thinner $(0.16 \mu \mathrm{m} /$ year $)$ with age. Our study also confirmed that the thickness of the peripapillary retinal nerve fiber decreased $0.93 \pm 4.24 \mu$ m over 6 months in the treatment group. The degree of decrease differs from the study by Parikh et al. ${ }^{5}$ because of sample differences. Many researchers, such as Lopes et al. ${ }^{6}$ and Takahashi et al., ${ }^{7}$ have suggested a relationship between diabetes and retinal nerve fiber loss. Ozdek et al. ${ }^{8}$ also suggested a strong relationship between diabetic retinopathy and retinal nerve fiber loss.

Chihara suggested that risk factors for RNFL defects in eyes with diabetes mellitus included a higher level of DR, systemic hypertension, and advanced age. However, visual acuity, disc size, axial length, and HBA1c level at the time of examination did not correlate with RNFL defects. ${ }^{9}$ In our study, the decrease in peripapillary RNFL thickness was more prominent when the blood HbAlc level was higher. In patients with diabetes, a high blood HbAlc suggests poor blood sugar control. When the blood sugar level is high there is an increased vulnerability to tissue viability, this in turn makes the retinal nerve fiber layer vulnerable to external insults, such as a laser.

In the treatment group, there was no relationship between changes in peripapillary RNFL thickness and changes in vision. The laser does not directly treat the fovea; therefore it is logical that the visual acuity was not affected.

PRP, which is used to treat diabetic retinopathy, lead to a decrease in peripapillary RNFL thickness in the treatment group. However, this decrease was not statistically significant when compared to the control group. The decrease was greater when the blood HbAlc level was higher. Based on this result, we suggest that the appropriate timing of PRP treatment may be determined after properly reducing the blood $\mathrm{HbA} 1 \mathrm{c}$ level if other conditions are tolerable.

One limitation of this study is that we only focused on 6 months of follow-up. Future studies should examine longterm results to determine if a similar pattern exists with longer follow-up.

\section{References}

1. Photocoagulation treatment of proliferative diabetic retinopathy. Clinical application of Diabetic Retinopathy Study (DRS) findings, DRS Report Number 8 . The Diabetic Retinopathy 
Study Research Group. Ophthalmology 1981;88:583-600.

2. Brinkmann R, Hüttmann G, Rögener J, et al. Origin of retinal pigment epithelium cell damage by pulsed laser irradiance in the nanosecond to microsecond time regimen. Lasers Surg Med 2000;27:451-64.

3. Tso MO, Wallow IH, Elgin S. Experimental photocoagulation of the human retina. I. Correlation of physical, clinical, and pathologic data. Arch Ophthalmol 1977;95:1035-40.

4. Brooks DE, Komàromy AM, Källberg ME.Comparative retinal ganglion cell and optic nerve morphology. Vet Ophthalmol 1999; 2:3-11.

5. Parikh RS, Parikh SR, Sekhar GC, et al. Normal age-related decay of retinal nerve fiber layer thickness. Ophthalmology
2007;114:921-6.

6. Lopes de Faria JM, Russ H, Costa VP. Retinal nerve fibre layer loss in patients with type 1 diabetes mellitus without retinopathy. Br J Ophthalmol 2002;86:725-8.

7. Takahashi H, Goto T, Shoji T, et al. Diabetes-associated retinal nerve fiber damage evaluated with scanning laser polarimetry. Am J Ophthalmol 2006;142:88-94.

8. Özdek S, Lonneville YH, Onol M, et al. Assessment of nerve fiber layer in diabetic patients with scanning laser polarimetry. Eye 2002;16:761-5.

9. Chihara E, Matsuoka T, Ogura Y, Matsumura M. Retinal nerve fiber layer defect as an early manifestation of diabetic retinopathy. Ophthalmology 1993;100:1147-51. 